\title{
The Effects of Computerized Gaming Program on Cognition in Children with Mental Retardation: A Case Study
}

\author{
Seon Chil Kim', Ju Young Heo², Hwa Kyung Shin², Byeong II Kim³ \\ ${ }^{1}$ Department of Biomedical Engineering, Keimyung University, Daegu; ${ }^{2}$ Department of Physical Therapy, Daegu Catholic University, Daegu; \\ ${ }^{3}$ Worisoft, Daegu, Korea
}

Purpose: The purpose of this study was to analysis of effectiveness between cognitive function assessment scores and gaming cognitive rehabilitation system in children with intellectual impairment.

Methods: Five children (male $=5$, age $=10.00 \pm 0.80$ ) with intellectual impairment participated in this study and were randomly assigned to the experiment that played (received) gaming cognitive rehabilitation system (Neuroworld). The children were applied 2 times a week for 30 minutes during 3 months. The children were assessed K-WSIC-VI (Korean-Wechsler intelligence scale for children-fourth edition) and recorded that gained score in gaming cognitive rehabilitation system before and after intervention. K-WSIC-VI contained five primary index scores: verbal comprehension index, visual spatial index, fluid reasoning index, working memory index, and processing speed index. Gaming cognitive rehabilitation system scoring was composed visual recall, target recall, sequence recall, selective attention, continuous attention, and exploration.

Results: In the intelligence quotient (IO) of K-WSIC-VI, there were significant increased in all children. The visual recall item was highest effective in all children. However, sequential recall showed the lowest improvement in all children. The performance speed of selective attention item was decreased, this means that children's skills have improved. Also, their ability to explore has improved significantly.

Conclusion: In conclusion, gaming cognitive rehabilitation system was significant effectiveness in cognitive function in some categories for children with intellectual impairment. However, the visual recall and performance speed don't represent of all cognitive function. Therefore, further studies will need to verify by applying more subject and longer duration.

Keywords: Cognitive function, Intellectual impairment, Gaming cognitive rehabilitation system

\section{INTRODUCTION}

Cognitive function is the skill of the cerebral cortex, which means the process of thinking, feeling and remembering the object of thinking or sensing. It can be deteriorated by numerous neural diseases or age which cause brain damage. Cognitive function is important factor to predict brain recovery. ${ }^{1}$ In general, cognitive processes are information processing processes that perceive, store, transform, and use information coming from the environment based on mental content such as thinking, reasoning, understanding, problem solving, and memory. ${ }^{2}$ Children with intellectual impairment has cognitive symptoms include memory, orientation, temporal and spatial cognition, judgment, abstract thinking, execu-

Received May 16, 2018 Revised Sep 21, 2018

Accepted Oct 25, 2018

Corresponding author Hwa Kyung Shin

E-mail hkshin0110@gmail.com tive functioning, and language ability impairment. ${ }^{3}$

According to previous studies analyzing the characteristics of the cognitive processes of children with intellectual disabilities, they showed a low concentration of attention due to lack of ability to allocate the state, ${ }^{4}$ sequential processing and simultaneous processing capability are lower than that of general children in coding process, ${ }^{5}$ Showed a defect in automated information processing. ${ }^{6}$ In addition, children with intellectual disabilities have visual deficits associated with most of the behaviors that occur in their daily lives, which is an important cause of learning difficulties. ${ }^{7}$ Besides it appears that there are deficiencies in the planning function, such as choosing an inefficient strategy for information processing, or difficulty maintaining and generalizing the strategy. ${ }^{8}$
Copylight ( 2018 The Korea Society of Physical Therapy

This is an Open Access article distribute under the terms of the Creative Commons Attribution Non-commercial License (Http:// creativecommons.org/license/by-nc/4.o.) which permits unrestricted non-commercial use, distribution, and reproduction in any medium, provided the original work is properly cited. 
The therapy for improving cognitive function has been widely used with traditional cognitive therapy as well as with computerized cognitive therapy. ${ }^{9}$ In particular, if we adopt routine computer games in our study, we can continue to do what we want to do in terms of training and learning more easily and for a longer period of time. In addition, very diverse forms of learning, ranging from physical to brain activity, can be carried out easily with low cost access. ${ }^{10}$ However, there has not yet been any validation of children with intellectual impairment, and there has been no validation with the test tools such as K-WSIC-VI, which can assess children's intelligence level besides MMSE. The purpose of this study was to investigate the effect of, gaming cognitive rehabilitation system, which can improve cognitive function, on cognitive ability of children with intellectual impairment.

\section{METHODS}

\section{Study design}

The present study was a case study research design. We compared the result pre and post. The reason we chose this research design is as follows. first, it is possible to the small number of participants can also be studied. It doesn't have to try to create groups that are alike on all important individual, environmental, and situational variables. Also, it can observe effects at the level of individual clients (the level at which we treat), and It can use when testing the intervention for the first time on a new population.

The design followed the guidelines for rigor and quality established for evidence level 5. This study incorporated "heterogeneous" participants with "low incidence" condition and participants that may "demonstrate variability from day to day"."

\section{Participants}

This study included 5 children with intellectual impairment. All 5 children were diagnosed with the 3 degree of disability. Children are referred to as child A, child B, child C, child D and child E to protect their identity (Table 1).

At the start of the study, child A (12 years, male) was diagnosed with the degree of disability when he was 7 years old. He has epilepsy and was taking medication. His LSSC (language scale for schoolaged children) score was 72 . The LSSC is a standardized language ability test for 700 children, from 106 to 123 students, taking into account regional distribution from grade 1 to grade 6 in elementary school. The validity of this was 0.88 and 0.87 in the comprehension and expression language test (RVET), and 0.70-0.90 in the K-WISC.

The internal consistency of the test items was 0.98 and the test retest reliability was $0.96 .^{12}$

Child B (10 years, male) was diagnosed with the degree of disability when he was 8 years old. He was not suffering from other complications. His LSSC score was 80. Child C (11 years, male) was diagnosed with the degree of disability when he was 9 years old. His LSSC was 71. Child D (10 years, male) and Child E (10 years, male) also was diagnosed with the degree of disability when they were 9 years old. Each LSSC score was 83 and 75. They hadn't particular complications. All of them has only cognitive impairments, not the genetic disease or syndrome.

Table 1. General characteristics of subjects

\begin{tabular}{|c|c|c|c|c|c|}
\hline & Child A & Child B & Child C & Child D & Child E \\
\hline Age (year) & 12.00 & 10.00 & 11.00 & 10.00 & 10.00 \\
\hline Gender (M/F) & M & $\mathrm{M}$ & M & M & M \\
\hline Height (cm) & 146.50 & 140.51 & 130.52 & 150.00 & 128.50 \\
\hline Weight (kg) & 36.01 & 37.00 & 32.11 & 34.00 & 31.00 \\
\hline \multicolumn{6}{|l|}{ History } \\
\hline Diagnosis & Cognitive impairment & Cognitive impairment & Cognitive impairment & Cognitive impairment & Cognitive impairment \\
\hline Age & 7.00 & 8.00 & 9.00 & 9.00 & 9.00 \\
\hline Complications & epilepsy & No & No & No & No \\
\hline Degree of disability & Grade 3 & Grade 3 & Grade 3 & Grade 3 & Grade 3 \\
\hline Intelligence quotient (IQ) & 66.00 & 67.00 & 65.00 & 67.00 & 66.00 \\
\hline Other assessment score (LSSC) & 72.00 & 80.00 & 71.00 & 83.00 & 75.00 \\
\hline
\end{tabular}

LSSC: language scale for school-aged children. 


\section{Gaming cognitive rehabilitation system for cognitive function} (Woorisoft, Korea)

Neuroworld (Woorisoft, Korea) is a suit of 6 mobile games, each of which is designed to address a particular aspect of cognitive functions. The 6 aspects include visual recall, target recall, sequence recall, selective attention, continuous attention, and exploration. The tested version can be installed in any Android smart phones and tablet computers (Figure 1). We applied interventions using tablet PC.

The children were applied 2times a week for 30 minutes during 3 months (24 times). The memory training included visual perception memory, object memory and sequential memory, each training time was 5 minutes. The attention training was also composed 15 minutes and It was selective attention, sustained attention and simultaneous coordination.

\section{Dependent valuable}

\section{1) K-WSIC-VI}

K-WISC-IV is an individual testing tool for assessing children's cognitive abilities from the age of 6 years to 16 years, 11 months. ${ }^{13}$ This allows a comprehensive assessment of overall cognitive function. It can distinguish between genius, intellectual impairment, and cognitive strengths and weaknesses. In this study, we selected this evaluation tool to identify detailed the cognitive abilities subtypes of children with intellectual disabilities. Various battery tests are car- ried out when diagnosing the disorder, but the most central view is the result of the K-WISC-IV. It is a standardized assessment tool designed to identify the ability of children with cognitive impairment.

\section{2) Gaming cognitive rehabilitation system for evaluation}

The game scoring included six subtypes was composed to evaluate various cognitive function. The visual recall means to perceive and memorize the position of things in visual space. The target recall refers to the process of storing and retrieving visual information, along with the ability to recall the major forms of stimulation. Sequence recall is the ability to recall the sequence of stimuli presented makes it possible to explain recall memory with visual memory. Selective attention refers to the ability to choose from various elements or stimuli present and focus on what is desired. ${ }^{14}$ Continuous attention is ability to focus on specific tasks over a period of time without confusion. ${ }^{14}$ Finally, exploration is the ability to handle more than one response or to respond to two or more different requests simultaneously. Basically, it is also called multitasking or dispatching by paying attention to more than one task (Figure 1). ${ }^{14}$ As the game progresses, if the player gets better, game level is more difficult. The Game recorded about correct answer time, success frequency and average score.
Visual recall

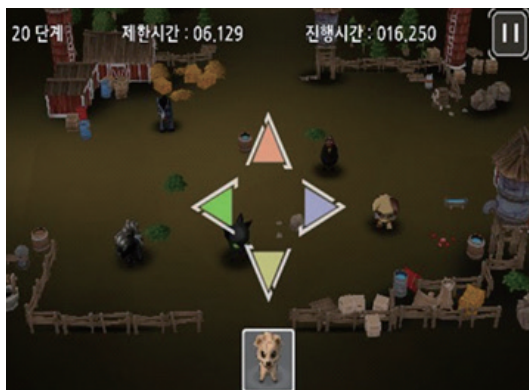

Selective attention

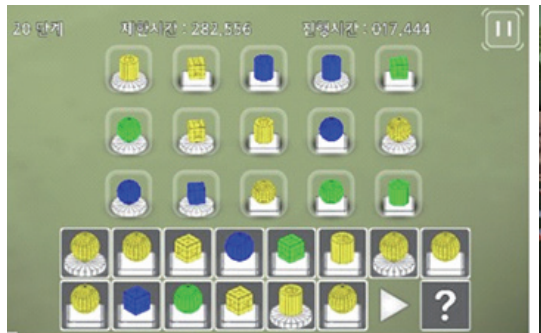

Target recall

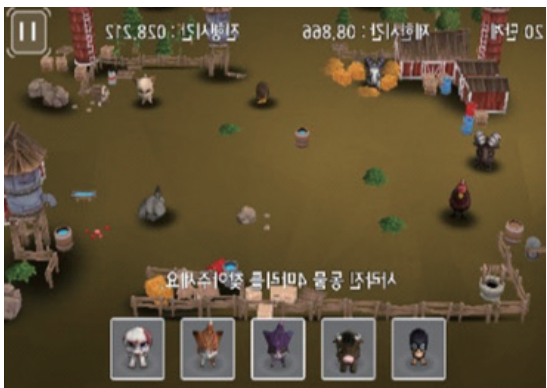

Continuous attention

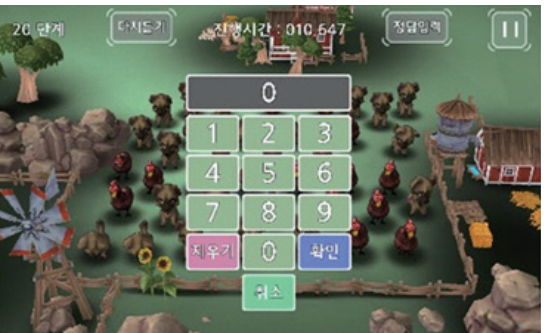

Sequence recall

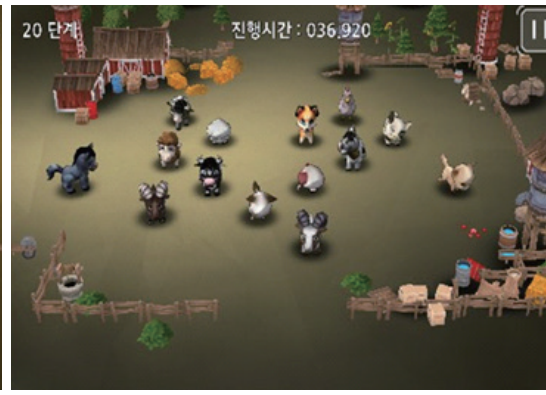

Exploration

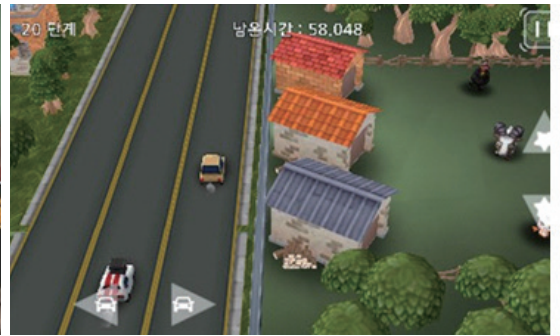

Figure 1. The gaming cognitive rehabilitation system. 


\section{Analysis}

We measured three times per subject with K-WSIC-VI and gaming cognitive rehabilitation system, and the average value was set as the representative value. The mean values of the gaming cognitive rehabilitation system were used as a representative value and compared pre and post (Tables 2, 3).

\section{RESULTS}

\section{K-WSIC-VI}

The children's IQ scores are significantly increased from before to after. The language comprehension item was increased in child B, C and D. The Perception reasoning item was increased except Child

Table 2. The result of K-WSIC-VI score before and after cognitive function game

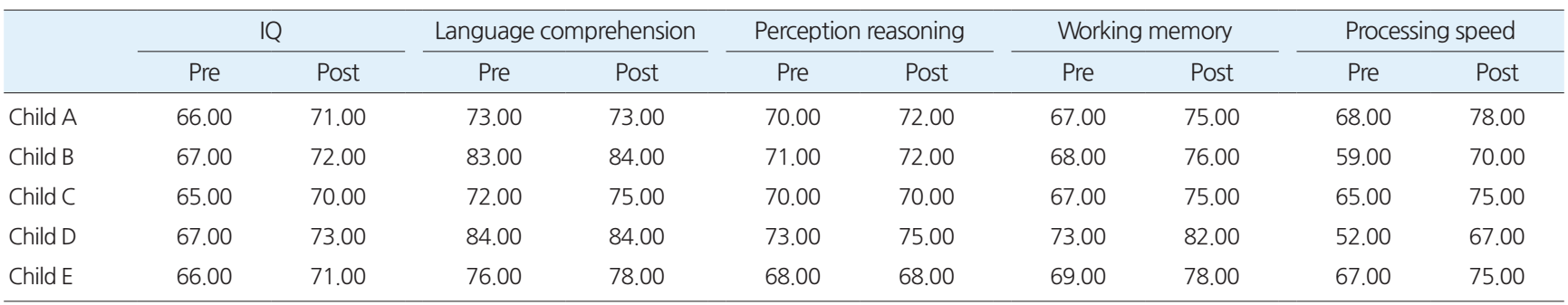

Table 3. The comparison of scores in before and after game (mean \pm SD)

\begin{tabular}{|c|c|c|c|c|c|c|c|c|c|}
\hline & & \multicolumn{2}{|c|}{ Level } & \multicolumn{2}{|c|}{ Enter correct answer (sec) } & \multicolumn{2}{|c|}{ Success frequency (\%) } & \multicolumn{2}{|c|}{ Average score } \\
\hline & & Pre & Post & Pre & Post & Pre & Post & Pre & Post \\
\hline \multirow[t]{7}{*}{ Child A } & Visual recall & 1.00 & 20.00 & 10.00 & 6.00 & 88.00 & 100.00 & 8.00 & 9.00 \\
\hline & Target recall & 1.00 & 19.00 & 9.00 & 6.00 & 25.00 & 73.00 & 8.00 & 9.00 \\
\hline & Sequence recall & 1.00 & 11.00 & 6.00 & 6.00 & 33.00 & 100.00 & 5.80 & 7.30 \\
\hline & Selective attention & 1.00 & 14.00 & 51.00 & 25.00 & 50.00 & 100.00 & 2.70 & 6.00 \\
\hline & Continuous attention & 1.00 & 20.00 & 13.00 & 3.00 & 48.00 & 35.00 & 6.00 & 8.00 \\
\hline & Exploration & 1.00 & 16.00 & 43.00 & 23.00 & 29.00 & 46.00 & 7.00 & 11.00 \\
\hline & Visual recall & 1.00 & 20.00 & 9.00 & 5.00 & 92.00 & 100.00 & 8.00 & 9.00 \\
\hline \multirow[t]{5}{*}{ Child B } & Target recall & 1.00 & 20.00 & 8.00 & 6.00 & 50.00 & 91.00 & 7.00 & 9.00 \\
\hline & Sequence recall & 1.00 & 8.00 & 5.00 & 8.00 & 33.00 & 50.00 & 6.40 & 7.30 \\
\hline & Selective attention & 1.00 & 20.00 & 6.00 & 53.00 & 50.00 & 100.00 & 2.70 & 7.00 \\
\hline & Continuous attention & 1.00 & 20.00 & 15.00 & 4.00 & 43.00 & 90.00 & 6.00 & 8.00 \\
\hline & Exploration & 1.00 & 16.00 & 42.00 & 26.00 & 43.00 & 55.00 & 7.00 & 12.00 \\
\hline \multirow[t]{6}{*}{ Child C } & Visual recall & 1.00 & 20.00 & 22.00 & 17.00 & 86.00 & 100.00 & 8.00 & 9.00 \\
\hline & Target recall & 1.00 & 19.00 & 10.00 & 4.00 & 75.00 & 33.00 & 7.00 & 9.00 \\
\hline & Sequence recall & 1.00 & 8.00 & 6.00 & 7.00 & 57.00 & 50.00 & 5.20 & 7.80 \\
\hline & Selective attention & 1.00 & 14.00 & 8.00 & 47.00 & 80.00 & 88.00 & 2.70 & 6.00 \\
\hline & Continuous attention & 1.00 & 20.00 & 2.00 & 7.00 & 56.00 & 40.00 & 6.00 & 7.00 \\
\hline & Exploration & 1.00 & 17.00 & 61.00 & 33.00 & 40.00 & 44.00 & 6.00 & 11.00 \\
\hline \multirow[t]{6}{*}{ Child D } & Visual recall & 1.00 & 20.00 & 9.00 & 6.00 & 92.00 & 100.00 & 7.00 & 9.00 \\
\hline & Target recall & 1.00 & 20.00 & 11.00 & 6.00 & 80.00 & 100.00 & 7.00 & 9.00 \\
\hline & Sequence recall & 1.00 & 8.00 & 6.00 & 5.00 & 50.00 & 50.00 & 6.70 & 7.80 \\
\hline & Selective attention & 1.00 & 18.00 & 21.00 & 38.00 & 67.00 & 100.00 & 2.90 & 7.90 \\
\hline & Continuous attention & 1.00 & 20.00 & 3.00 & 8.00 & 78.00 & 67.00 & 6.00 & 7.00 \\
\hline & Exploration & 1.00 & 18.00 & 43.00 & 19.00 & 43.00 & 47.00 & 5.00 & 11.00 \\
\hline \multirow[t]{6}{*}{ Child E } & Visual recall & 1.00 & 20.00 & 1.00 & 6.00 & 93.00 & 100.00 & 7.00 & 10.00 \\
\hline & Target recall & 1.00 & 20.00 & 10.00 & 5.00 & 75.00 & 58.00 & 7.00 & 9.00 \\
\hline & Sequence recall & 1.00 & 6.00 & 5.00 & 8.00 & 33.00 & 33.00 & 6.10 & 7.60 \\
\hline & Selective attention & 1.00 & 17.00 & 8.00 & 25.00 & 33.00 & 50.00 & 2.00 & 6.20 \\
\hline & Continuous attention & 1.00 & 20.00 & 12.00 & 4.00 & 48.00 & 95.00 & 7.00 & 8.00 \\
\hline & Exploration & 1.00 & 17.00 & 38.00 & 26.00 & 63.00 & 46.00 & 6.00 & 11.00 \\
\hline
\end{tabular}


$\mathrm{C}$ and $\mathrm{E}$. The Working memory item was significantly increased in all child.

Child A working memory was increased from 67 to 75 . Child B working memory was increased from 68 to 76 . Child C's working memory was increased from 67-75. Working memory of child D was increase from 73 to 82 . Child E working memory item was increased from 69-78. Processing speed also increased in all child. Child A's processing speed was increased from 69 to 78 . Child B's processing speed was increased from 59 to 70 . Child C was increased from 65 to 75 . Child D was increased from 52 to 67 . The child E was increased from 67 to 75 (Table 2).

\section{Game score}

The visual recall items were significant increased in all child. Their level score were upped from 1 to 20. The target recall items were increased in child B, D and E. Their level score were upped from 1 to 20. The child A and C were increased from 1 to 19 level. The sequence recall items were lower than visual recall and target recall. The child B, C and D were increased from 1 to 8 level. The child D was increased in 5 level. Child A was most improved from 1 to 11 level. The selective attention items were improved for all children. Child B was most improved from 1 to 20 level, child A and C were the lowest improvement from 1 to 14 level. The continuous attention items were improved from 1 to 20 level for all children. The exploration of child A and B were improved from 1 to 20 level. Child $\mathrm{C}$ and $\mathrm{E}$ were increased from 1 to 17 level. Child D was rose from 1 to 18 the most. As the input speed of entering the correct answer decreases, it means improvement of cognitive function. All of the items were speeded up and improved, child B's sequence recall and selective attention were slowed down and he's skills decreased. Also, child C's sequence recall, selective attention and continuous attention item were slowed down. Likewise, selective attention and continuous attention of child D were slowed down. Child D's visual recall was decreased from 1 to 6 . Sequence recall was increased from 5 to 8 . Selective attention also was expressed increasing speed from 8 to 25 . The frequency of success means the number of successful per task. It means that the higher the percentage, the better the skill. The success rate for each child has increased, but some areas have shown a tendency to decline. In case of child A, he decreased from 48 to $35 \%$ in continuous attention item. But success rate of child B was significant increased in all items. Child $\mathrm{C}$ was decreased in tar- get recall, sequence recall and continuous recall. Child D's continuous attention item were decreased from 78 to $67 \%$. In child $\mathrm{E}$, target recall, sequence recall and exploration were decreased in each items (Table 3).

\section{DISCUSSION}

In this study, we investigated the effect of gaming cognitive rehabilitation system in children with intellectual impairment. Cognitive function refers to intellectual ability such as memory, orientation, judgment, attention, calculation ability, and language ability. The area of the brain that is most related to cognitive function is the part of the frontal lobe, which performs executive functions such as attention and control, and is involved in the selection, initiation, and suppression of motor reactions. ${ }^{15}$ Intellectual impairment is a group of children with cognitive problems, When the function of the frontal lobe is decreased, the ability to control basic cognitive functions such as attention, language, time and space, and memory is reduced. ${ }^{16}$ In this study, we observed the improvement of cognitive function by applying the game program to 5 children with intellectual impairment for 3 months. All five children showed an improved level of post-test comparisons in the K-WSIC-VI, which improved on all of the following items: verbal comprehension index, visual spatial index, fluid reasoning index, working memory index, and processing speed index. Previous studies have shown that computerized cognitive training was significantly effective when applied to patients with mild dementia. ${ }^{17}$ When applying Contras, a computerized cognitive program, to children with intellectual impairment, the child's visual perception and daily life skills are improved. ${ }^{18}$

On the other hand, the gaming cognitive rehabilitation system applied to this study is structured to evaluate six detailed items. Visual recall was improved in all children from level 1 to level 20, the correct answer time was shortened except for children D. Also, it was confirmed that the frequency of success was increased to $100 \%$. Thus, the most effective enhancement of the cognitive function program is visual memory. The next highest level of improvement was the target recall. The performance of children A and C was elevated to 19 levels and the rest of the children rose to 20 levels. The performance speed of the target recall was decreased, indicating that the task performance was improved. In the case of child A, which showed the highest level of progressive memory, the level of 
the sequential recall was 11 , and the other children increased by 6 to 8. In addition, sequential recall did not increase except for A children with increased frequency from 33 to $100 \%$ and B children from 33 to $50 \%$. This is probably the most difficult item for children when compared to other items. The children who reached the highest performance level in the selective attention item were 20 (child B), and the children who reached the lowest performance level were 14. However, except for the child A (51-25seconds decreased), the response time was longer and the frequency of success was higher. This means that even though the execution time is longer, it is better to perform the task accurately. Continuous attention was the most necessary item for concentration, but the performance level increased only child B and D. Finally, exploration was raised to 16 for children A\&B, 17 for children C\&E, and 18 for child D. Exploration items increased the speed of all children, and the frequency of success was also increased except for child D.

Therefore, in this study, the highest rate of increase in all children in the gaming cognitive rehabilitation system score item was visual recall item and exploration. The lowest rate of improvement was sequence recall. And the next difficult item was constant attention. Therefore, it was confirmed through this study that the game program was effective in improving the cognitive function of the child, and the visual memory which quickly memorized one of the game items was the most effective. The children had the most difficult sequential recall that they had to remember a lot. This suggests that sequential memory items and selective attention items require more cognitive functions than simple visual items. This study has proved that the game is effective for the visual perception ability of children. When a pointing game using a computer was applied to an intellectual impairment child, it was improved visual perceptual capabilities.10 Therefore, it is considered that computer games are effective to improve visual perception ability of children with intellectual impairment. However, this study has some limitation. The visual recall and performance speed don't represent of all cognitive function. Therefore, further studies will need to verify by applying more subject and longer duration. Also, this study applied the game program to children with intellectual impairment improve cognitive function but did not evaluate interest in game program characteristics. Thus, it is considered necessary for future studies to also prove the effects on the inner motivations and interests of children, which are the advantages of game programs.

\section{REFERENCES}

1. Lee SR, Kwon HC. The relationship between activities of daily living and cognitive score in stroke patients. Phys Ther Korea. 2003;10(3):41-51.

2. Ko MJ, Kang YS. Comparison on cognitive function of children with and without intellectual disabilities using CAS. 2011;13(4):41-9.

3. Bullock R, Hammond G. Realistic expectations: the management of severe Alzheimer disease. Alzheimer Dis Assoc Disord. 2003;17:S80-5.

4. Melnyk L, Das J. Measurement of attention deficit: correspondence between rating scales and tests of sustained and selective attention. Am J Ment Retard. 1992;96(6):599-606.

5. Kim EK. The cognitive characteristics of 3-6 years-old children with SLI, mental retardation, cerebral palsy based on the K-ABC. Korean Journal of Early Childhood Education. 2004;4:53-69.

6. Jo YT. A study on automatic process defects of tasks in short-term memory of mental retarded children. The Korean Society of Special Education. 1994;15:73-87.

7. Hong JR. Effect of program in visual perception on eye-hand coordination in a child with cerebral palsy: a case study. J Korean Soc Occup Ther. 2000;8(1):103-10.

8. Kittler P, Krinsky-McHale S, Devenny D. Sex differences in performance over 7 years on the Wechsler intelligence scale for children-revised among adults with intellectual disability. J Intellect Disabil Res. 2004; 48(2):114-22.

9. Cho YN. The effects of computerized cognitive rehabilitation on cognitive function in elderly post-stroke patients. Daegu University. Dissertation of Doctorate Degree. 2010.

10. Kim KJ, Kim SW. Effects of computer pointing game on visual perception ability of children with intellectual disability. J Korea Soc Comput Inf. 2010;18(2):449-52.

11. Logan LR, Hickman RR, Harris SR et al. Single-subject research design: recommendations for levels of evidence and quality rating. Dev Med Child Neurol. 2008;50(2):99-103.

12. Choi J, Oh SJ, Lee Y. Language characteristics of school-aged children with high-functioning ASD through the LSSC. Special Education. 2015;14(2):139-56.

13. Kwak KJ, Oh SW, Kim CT. Expert guidline of K-WISC- VI. Seoul, Hakjisa, 2011.

14. Cohen RA, Sparling-Cohen YA, O'Donnell BF. The neuropsychology of attention. New York, Springer, 1993.

15. Park R, Lee H, Kim S. Correlation analysis between MBI and MMSE after exercise program for dementia elderly. J Kor Phys Ther. 2000;12:8393.

16. Park TJ. Cognitive neural mechanisms of aging. Korean J Exp Psychol. 2004;16(16):317-36.

17. Kang JH. Effect of computerized cognitive training on cognitive function, instrumental activities of daily living and quality of life in patients with mild dementia. The Journal of Korea Aging Friendly Industry Association. 2016;8(2):71-83.

18. Kim SY, Lee KM. The effect of computer-based cognitive rehabilitation program on visual perception and ADL in children with intellectual disability. RESKO. 2015;9(2):105-13. 